\title{
A detailed anatomical and mathematical model of the hippocampal formation for the generation of Sharp-Wave Ripples and Theta-nested Gamma oscillations
}

\author{
Amélie Aussel • Laure Buhry • Louise \\ Tyvaert - Radu Ranta
}

Pre-print version. The published version can be found at https://doi.org/10.1007/s10827-0180704-x

\begin{abstract}
The mechanisms underlying the broad variety of oscillatory rhythms measured in the hippocampus during the sleep-wake cycle are not yet fully understood. In this article, we propose a computational model of the hippocampal formation based on a realistic topology and synaptic connectivity, and we analyze the effect of different changes on the network, namely the variation of synaptic conductances, the variations of the CAN channel conductance and the variation of inputs. By using a detailed simulation of intracerebral recordings, we show that this model is able to reproduce both the theta-nested gamma oscillations that are seen in awake brains and the sharp-wave ripple complexes measured during slow-wave sleep. The results of our simulations support the idea that the functional connectivity of the hippocampus, modulated by the sleep-wake variations in Acetylcholine concentration, is a key factor in controlling its rhythms.
\end{abstract}

Keywords Hippocampal oscillations · Conductance-based neurons · Sleep-wake cycle $\cdot$ Sharp-Wave Ripples · Theta-nested gamma oscillations · Acetylcholine

\section{Introduction}

The hippocampus can exhibit different oscillatory rhythms within the sleep-wake cycle, each of them being involved in cognitive processes. For example, theta-nested gamma oscillations, consisting of the coupling of theta $(4-12 \mathrm{~Hz})$ and gamma $(40-$ $100 \mathrm{~Hz}$ ) rhythms, are produced during wakefulness and are associated with spatial

A. Aussel E-mail: amelie.aussel@loria.fr

A. Aussel · L. Buhry

Laboratoire Lorrain de Recherche en Informatique et ses applications (LORIA UMR 7503, University of Lorraine-INRIA-CNRS, F-54506)

A. Aussel · L. Tyvaert · R. Ranta

Centre de Recherche en Automatique de Nancy (CRAN-CNRS UMR 7039 University of Lorraine, FRANCE)

L. Tyvaert

Department of Neurology, CHU de Nancy, Nancy, FRANCE. 
navigation and working memory tasks ([O'Keefe and Recce, 1993], [Axmacher et al., 2010]), whereas Sharp-Wave-Ripple (SWR) complexes, consisting of fast $(140-200 \mathrm{~Hz})$ oscillatory events occurring during low frequency waves $(\leq 0.5 \mathrm{~Hz})$, are produced during slow-wave sleep and quiet waking and play an important role in memory consolidation ([Girardeau and Zugaro, 2011], [Buzsáki, 2015]). Models exist to reproduce and explain the generation of each of these rhythms individually: for Sharp-Wave Ripples, see [Taxidis et al., 2012] or [Traub and Bibbig, 2000] and for theta-nested gamma oscillations, see [Pastoll et al., 2013], [Fukai, 1999], or [Bartos et al., 2007]. However, to the best of our knowledge, there is at the moment no model capable of generating both rhythms and functionally switching between them.

The transitions between these rhythms suggest a change in the functional connectivity of the hippocampus. Some authors ([Hasselmo, 1999], [Platt and Riedel, 2011], see also [Tiesinga et al., 2001]) propose that this phenomenon may be due to a neuromudulator, Acetylcholine (Ach), whose concentration is higher during active wakefulness than sleep, modulating synaptic transmissions. Ach concentration can also vary rapidly during wakefulness (see [Ruivo et al., 2017] and [Sarter et al., 2009] for studies in rodents), and therefore it could also possibly account for the SWRs that are seen in quiet awake individuals ([Foster and Wilson, 2006]). But though we understand the influence of Ach on individual cells for different receptor types and locations ([Drever et al., 2011]), its quantitative effects on the whole hippocampal network remain unclear.

In this context, we have built a computational model of the hippocampal formation that exhibits characteristic rhythms of wakefulness and slow-wave sleep, considering the varying concentration of Ach. In order to simulate the complete hippocampal formation, our model uses point neural models (single-compartment) but having realistic dynamics (conductance-based Hodgkin-Huxley neurons). Among these neurons, some have one of the membrane channel's conductance directly linked to the level of Ach (CAN, see [Giovannini et al., 2017]). The microscopic anatomy of the neurons was approximated by two compartments (the simplest shape able to generate a relatively realistic LFP through a dipolar electric field [Pettersen et al., 2012]), while the macroscopic anatomy of the hippocampal structure was reproduced by positioning and connecting the neurons in an anatomically realistic manner. Based on the model proposed in [Hasselmo, 1999], the network's functional connectivity was changed between wakefulness and slow-wave sleep. Moreover, the stimulation entry of the network was derived from real sEEG measurements recorded during wake/sleep in the human prefrontal cortex (projecting on the entorhinal cortex). In order to compare our results with in vivo signals from the human hippocampus, we also simulated the signals recorded by a realistic macroscopic sEEG electrode placed within the network.

Our main finding is that our model can indeed reproduce both theta-nested gamma oscillations and SWR complexes in humans by changing the level of Ach, with but little influence of the input stimulus. The network connectivity seems to determine the high frequency component of the rhythms, whereas individual neurons channel conductance seem to determine its low frequency component.

In the following section, we describe our model in terms of individual neuron equations, synapses, topology, and electrode simulation, and we highlight the connectivity parameters that were changed to represent slow-wave sleep and wakefulness. We then present and analyze the oscillatory patterns we obtain. 


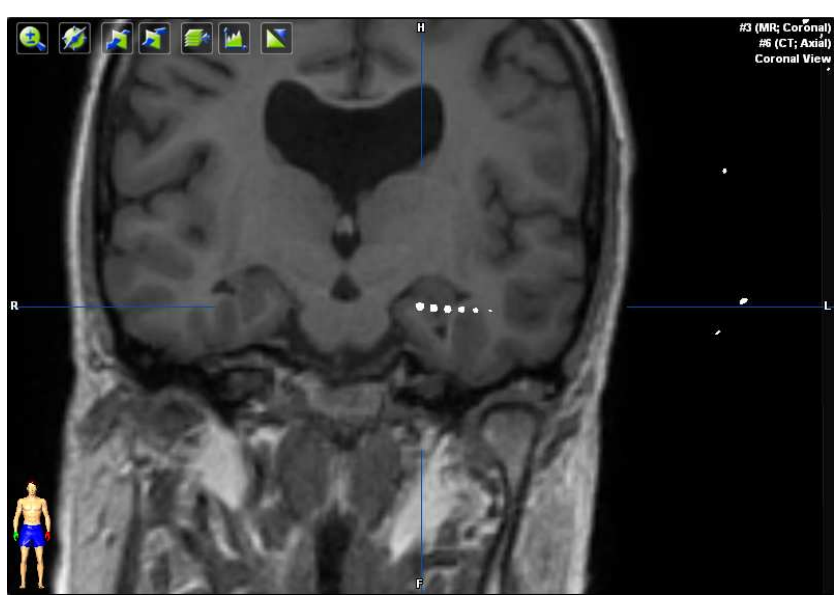

Fig. 1 Coregistered CT-MRI image of the implantation of an SEEG electrode in the patient's hippocampus (coronal view).

\section{Materials and Methods}

\subsection{Experimental data}

The LFP data we used was obtained from a patient suffering from refractory epilepsy, implanted with intracranial electrodes for surgery planning at the Neurology Service of the University Hospital (CHU) from Nancy, France. The patient gave his informed consent and the study was approved by the ethics committee of the hospital. The SEEG electrodes (Dixi Medical@, France) had a diameter of $0.8 \mathrm{~mm}$, with $2 \mathrm{~mm}$-long contacts and $1.5 \mathrm{~mm}$ inter-contact distance. The patient was notably implanted in the prefrontal cortex, the temporal lobe and the hippocampus (see Figure 1 for an MRI image of the implatation of an electrode in the hippocampus), but the epileptogenic zone was located in another region. The position of the electrodes in the patient's brain was automatically ascertained using a procedure described in [Hofmanis et al., 2011].

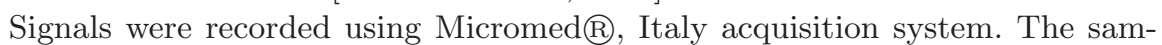
pling frequency was $1024 \mathrm{~Hz}$. All signals were labelled by a neurologist to identify the different stages of the sleep-wake cycle (based on EEG signal) as well as to exclude any epileptic phenomena from the data (interictal or seizures). Therefore we assumed that, when no seizure or epileptic spike occurred, the signals from these regions were similar to that of healthy people. We used signals from wakefulness and slow-wave sleep states.

\subsection{Computational model}

\subsubsection{Neuron dynamics models}

The neurons in our network were simulated using a single compartment conductancebased model derived from Hodgkin-Huxley's ([Hodgkin and Huxley, 1952]). The 
temporal evolution of each neuron membrane potential $V_{m}$ followed a differential equation of the form:

$$
C_{m} \frac{d V_{m}}{d t}=-I_{\text {leak }}-\sum I_{\text {channel }}-I_{\text {syn }}-I_{\text {syn }}+\eta
$$

where $I_{\text {leak }}$ is a leakage current, each $I_{\text {channel }}$ is the membrane current associated with the ion channel channel, $I_{s y n_{E}}$ and $I_{s y n_{I}}$ are the excitatory and inhibitory synaptic input to the cell, respectively, and $\eta$ is a random gaussian noise accounting for unknown entries.

Our principal excitatory neurons were pyramidal cells modelled with the following ion channels: fast sodium $\left(I_{N a}\right)$, potassium $\left(I_{K}\right)$, low-threshold calcium current $\left(I_{C a}\right)$, and potassium M-current $\left(I_{M}\right)$. Some pyramidal cells were also added a Calcium-Activated-Nonspecific (CAN) cationic channel, with corresponding current $I_{C A N}$. In the following, these two types of pyramidal cells are referred to as non-CAN and CAN respectively. Interneurons were modelled with the following ion channels : fast sodium $\left(I_{N a}\right)$, and potassium $\left(I_{K}\right)$.

The complete expressions for all these ionic channel currents are detailed in [Giovannini et al., 2017].

The leakage currents obey the following equation :

$$
I_{\text {leak }}=\left(g_{\text {leak }} \times A\right) \times\left(V_{m}-E_{\text {leak }}\right)
$$

where $g_{\text {leak }}$ is the maximum leaking conductance, $A$ is the area of the neuron membrane and $E_{\text {leak }}$ is the channel reversal potential.

The $I_{K}, I_{M}$ and $I_{C A N}$ currents follow a set of equations of the form :

$$
I_{\text {channel }}=\left(g_{\text {channel }} \times A\right) \times m^{k} \times\left(V_{m}-E_{\text {channel }}\right)
$$

where $m$ is a gating variable defined by :

$$
\frac{d m}{d t}=\frac{m_{\infty}-m}{\tau_{m}}
$$

For $I_{K}$ and $I_{C A N}$, the $m_{\infty}$ and $\tau_{m}$ parameters depend on two functions $\alpha_{m}$ and $\beta_{m}$ :

$$
\begin{aligned}
m_{\infty} & =\frac{\alpha_{m}}{\alpha_{m}+\beta_{m}} \\
\tau_{m} & =\frac{0.2}{\alpha_{m}+\beta_{m}}
\end{aligned}
$$

Similarly, the $I_{N a}$ and $I_{C a}$ currents follow a set of equations of the form :

$$
I_{\text {channel }}=\left(g_{\text {channel }} \times A\right) \times m^{k} \times n \times\left(V_{m}-E_{\text {channel }}\right)
$$

with two gating variables $\mathrm{m}$ and $\mathrm{n}$, such as :

$$
\begin{aligned}
\frac{d m}{d t} & =\frac{m_{\infty}-m}{\tau_{m}} & \frac{d n}{d t} & =\frac{n_{\infty}-n}{\tau_{n}} \\
m_{\infty} & =\frac{\alpha_{m}}{\alpha_{m}+\beta_{m}} & n_{\infty} & =\frac{\alpha_{n}}{\alpha_{n}+\beta_{n}} \\
\tau_{m} & =\frac{0.2}{\alpha_{m}+\beta_{m}} & \tau_{n} & =\frac{0.2}{\alpha_{n}+\beta_{n}}
\end{aligned}
$$


Table 1 Parameter values of interneurons

\begin{tabular}{|l|c|}
\hline Parameter & Expression \\
\hline$A$ & $14.10^{3} \mathrm{~cm}^{2}$ \\
$g_{\text {leak }}$ & $0.1 \mathrm{mS} / \mathrm{cm}^{2}$ \\
$E_{\text {leak }}$ & $-90 m V$ \\
$g_{K}$ & $9 m S / \mathrm{cm}^{2}$ \\
$E_{K}$ & $-65 m V$ \\
$k_{K}$ & 4 \\
$\alpha_{m, K}$ & $0.01 \frac{V_{M}+34 m V}{1-e^{-0.1\left(V_{M}+34 m V\right)}}$ \\
$\beta_{m, K}$ & $0.125 e^{-\frac{V_{M}+44 m V}{80 m V}}$ \\
$g_{N a}$ & $35 m S / c m^{2}$ \\
$E_{N a}$ & $55 m V$ \\
$k_{N a}$ & 3 \\
$\alpha_{m, N a}$ & $0.1 \frac{V_{M}+35 m V}{1-e^{-0.1\left(V_{M}+35 m V\right)}}$ \\
$\beta_{m, N a}$ & $4 e^{-\frac{V_{M}+60 m V}{18 m V}}$ \\
$\alpha_{n, N a}$ & $0.07 e^{-\frac{V_{M}+58 m V}{20 m V}}$ \\
$\beta_{n, N a}$ & $\frac{1}{e^{1-0.1 *\left(V_{M}+28 m V\right)}}$ \\
\hline
\end{tabular}

Finally the gating variable of $I_{C A N}$ depends on the calcium concentration inside the neuron, $[\mathrm{Ca}]_{i}^{2+}$, defined by :

$$
\begin{aligned}
\frac{d[\mathrm{Ca}]_{i}^{2+}}{d t} & =\gamma\left(I_{C a}\right)+\frac{\left([\mathrm{Ca}]_{\infty}^{2+}-[\mathrm{Ca}]_{i}^{2+}\right)}{\tau_{[\mathrm{Ca}]^{2+}}} \\
\gamma\left(I_{C a}\right) & =\frac{-k_{u} \times I_{C a}}{2 \times F \times \operatorname{depth} \times A}
\end{aligned}
$$

where $\tau_{[\mathrm{Ca}]^{2+}}=1 \mathrm{~s}$ represents the rate of calcium removal from the cell, $[\mathrm{Ca}]_{\infty}^{2+}=$ $0.24 \mathrm{~mol} / L$ is the calcium concentration if the calcium channel remains open for a duration $\Delta_{T} \rightarrow \infty, k_{u}=10^{4}$ is a unit conversion constant, $F$ is Faraday's constant and depth $=1 \mu \mathrm{m}$ is the depth at which the calcium is stored inside the cell.

The amplitude of the noise $\eta$ (Equation 1) simulating random unknown inputs to our network was set to $500 \mathrm{pA}$.

The full expression of all the parameters defined here can be found in Table 1 and Table 2 for interneurons and pyramidal neurons, respectively.

\subsubsection{Synaptic models}

The interactions between neurons were modelled as AMPA and GABA-A synapses, with $I_{s y n_{E}}$ and $I_{s y n_{I}}$ currents for excitatory and inhibitory connections respectively. These currents are described by the following bi-exponential differential equations:

$$
\begin{aligned}
I_{s n_{I, E}} & =g_{I, E}\left(V_{m}-E_{I, E}\right), \\
\frac{d g_{I, E}}{d t} & =\left(-g_{I, E}+h_{I, E}\right) \frac{1}{\tau_{g_{I, E}}}, \\
\frac{d h_{I, E}}{d t} & =-h_{I, E} \frac{1}{\tau_{h_{I, E}}} .
\end{aligned}
$$


Table 2 Parameter values of pyramidal neurons

\begin{tabular}{|c|c|}
\hline Parameter & Expression \\
\hline$A$ & $29.10^{3} \mathrm{~cm}^{2}$ \\
\hline$g_{\text {leak }}$ & $0.01 \mathrm{mS} / \mathrm{cm}^{2}$ \\
\hline$E_{\text {leak }}$ & $-70 \mathrm{mV}$ \\
\hline$g_{K}$ & $5 \mathrm{mS} / \mathrm{cm}^{2}$ \\
\hline$E_{K}$ & $-100 m V$ \\
\hline$k_{K}$ & 4 \\
\hline$\alpha_{m, K}$ & $-0.032 \frac{V_{M}+40 m V}{e^{-1-0.2\left(V_{M}+40 m V\right)}}$ \\
\hline$\beta_{m, K}$ & $0.5 e^{-\frac{V_{M}+45 m V}{40 m V}}$ \\
\hline$g_{N a}$ & $50 \mathrm{mS} / \mathrm{cm}^{2}$ \\
\hline$E_{N a}$ & $50 \mathrm{mV}$ \\
\hline$k_{N a}$ & 3 \\
\hline$\alpha_{m, N a}$ & $-0.32 \frac{V_{M}+42 m V}{e^{-\frac{V_{M}+42 m V}{4 m V}}-1}$ \\
\hline$\beta_{m, N a}$ & $0.28 \frac{V_{M}+15 m V}{e^{-\frac{V_{M}+15 m V}{5 m V}}-1}$ \\
\hline$\alpha_{n, N a}$ & $0.128 e^{-\frac{V_{M}+38 m V}{18 m V}}$ \\
\hline$\beta_{n, N a}$ & $\frac{4}{1+e^{-\frac{V_{M}+15 m V}{5 m V}}}$ \\
\hline$g_{M}$ & $90 \mu \mathrm{S} / \mathrm{cm}^{2}$ \\
\hline$E_{M}$ & $-100 m V$ \\
\hline$k_{M}$ & 1 \\
\hline$m_{\infty, M}$ & $\frac{1}{1+e^{-\frac{V_{M}+35 m V}{10 m V}}}$ \\
\hline$\tau_{m, M}$ & $\frac{1}{3.3 e^{\frac{V_{M}+35 m V}{20 m V}}+e^{-\frac{V_{M}+35 m V}{20 m V}}}$ \\
\hline$g_{C a}$ & $0.1 \mathrm{mS} / \mathrm{cm}^{2}$ \\
\hline$E_{C a}$ & $120 m V$ \\
\hline$k_{C a}$ & 2 \\
\hline$\alpha_{m, C a}$ & $-0.055 \frac{V_{M}+27 m V}{e^{-\frac{V_{M}+27 m V}{3.8 m V}}-1}$ \\
\hline$\beta_{m, C a}$ & $-0.94 e^{\frac{V_{M}+75 m V}{17 m V}}$ \\
\hline$\alpha_{n, C a}$ & $-0.000457 e^{\frac{V_{M}+13 m V}{50 m V}}$ \\
\hline$\beta_{n, C a}$ & $\frac{0.0065}{e^{-\frac{V_{M+15 m V}}{28 m V}}+1}$ \\
\hline$g_{C A N}$ & $25 \mu \mathrm{S} / \mathrm{cm}^{2}$ \\
\hline$E_{C A N}$ & $-20 m V$ \\
\hline$k_{C A N}$ & 2 \\
\hline$\alpha_{m, C A N}$ & $0.0002 \cdot 3^{1.4} \frac{[\mathrm{Ca}]_{i}^{2+}}{0.5 \mathrm{~mol} / \mathrm{L}}$ \\
\hline$\beta_{m, C A N}$ & $0.0002 \cdot 3^{1.4}$ \\
\hline
\end{tabular}

Table 3 Parameter values of the biexponential synapses

\begin{tabular}{|l|c|c|}
\hline Synapse Type & AMPA & GABA \\
\hline Resting Potential $(\mathrm{E})$ & $0 \mathrm{mV}$ & $-80 \mathrm{mV}$ \\
\hline Time constant for rise $\left(\tau_{g}\right)$ & $0.3 \mathrm{~ms}$ & $1 \mathrm{~ms}$ \\
\hline Time constant for decay $\left(\tau_{h}\right)$ & $5 \mathrm{~ms}$ & $10 \mathrm{~ms}$ \\
\hline Maximum Conductance $\left(g_{\max }\right)$ & $60 \mathrm{pS}$ & $600 \mathrm{pS}$ \\
\hline
\end{tabular}

Whenever a pre-synaptic spike occurs, the value of $g_{I}$ or $g_{E}$ in the post-synaptic neuron is increased by a fixed amount $g_{\max _{E}}$ or $g_{\max _{I}}$.

The values of the synaptic resting potentials $E_{I}$ and $E_{E}$, and the synaptic time constants of rise and decay $\tau_{g_{E}}, \tau_{h_{E}}, \tau_{g_{I}}$ and $\tau_{h_{I}}$ are summed up in Table 3. 
Table 4 Number of excitatory neurons $\left(N_{E}\right)$ and interneurons $\left(N_{I}\right)$ in each region

\begin{tabular}{|l|c|c|}
\hline Region & $N_{E}$ & $N_{I}$ \\
\hline Entorhinal cortex & 10000 & 1000 \\
\hline Dentate Gyrus $\left(\tau_{g}\right)$ & 10000 & 100 \\
\hline CA3 $\left(\tau_{h}\right)$ & 1000 & 100 \\
\hline CA1 $\left(g_{\max }\right)$ & 10000 & 1000 \\
\hline
\end{tabular}

\subsubsection{Anatomical modelling}

At the macroscopic level, our network is composed of two different structures: one representing the hippocampus, and the other representing the entorhinal cortex. The hippocampus itself is divided into three substructures: the dentate gyrus, CA3 and CA1. A 10:1 ratio between pyramidal neurons and interneurons, as reported in [Jinno and Kosaka, 2010] for dorsal hippocampus, was respected in all regions but the dentate gyrus. The same 10:1 ratio was used between the total number of neurons in CA1 and the total number of neurons in CA3, as reported in humans in [West and Gundersen, 1990].

As for the dentate gyrus, we considered a ratio 100:1 ratio between the pyramidal cells and the interneurons, because of the higher proportion of excitatory cells compared to inhibitory cells in this structure ([Patton and McNaughton, 1995],[Jinno and Kosaka, 2010]). The number of excitatory cells as well as interneurons are summed up in Table 4. CAN pyramidal neurons were used in the entorhinal cortex, CA3 and CA1, while non-CAN neurons were used in the dentate gyrus.

As our goal is to model realistically the population's extracellular recordings, the position of the entorhinal cortex and the hippocampal structures and the different types of neurons within them were chosen in accordance with human anatomy. A $15 \mathrm{~mm}$-thick layer of the hippocampal formation was reproduced in this way, with pyramidal neurons uniformly distributed within the stratum pyramidale (or within the stratum granulosum for the dentate gyrus), and interneurons uniformly distributed within the stratum oriens. For each pyramidal neuron, its projection on the stratum moleculare was also computed as an estimation of the position of its apical dendrites (Figure 2), and thus the orientation of the (neural) elementary dipole used for generating the extracellular recordings. In other words, at the cellular level, the detailed geometry of the pyramidal neurons was simplified to simple current dipoles [Pettersen et al., 2012]. Moreover, as interneurons contributions to the LFP are very small [Mazzoni et al., 2015], their microscopic geometry was neglected (point neurons).

\subsubsection{Structural connectivity}

As it is commonly accepted that the hippocampal formation receives most of its external inputs through the entorhinal cortex, only these neurons receive external stimulation in our model ([Andersen et al., 2007]). From this region, the neural activity propagates in the hippocampus through the so-called "tri-synaptic way", going from the entorhinal cortex to the dentate gyrus, from the dentate gyrus to CA3, from CA3 to CA1, and finally from CA1 back to the entorhinal cortex. Fewer connections are also made from the entorhinal cortex directly to CA3 and 


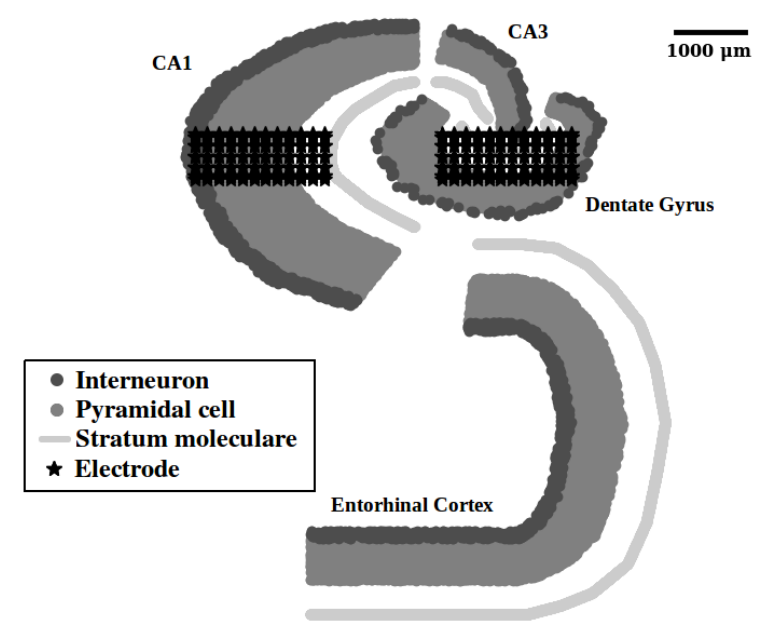

Fig. 2 Topology of the entorhinal cortex and the hippocampus used in the model

Table 5 Probability of the synaptic connectivity between each region. The same value is used whether the target cells are pyramidal neurons or interneurons. EC: Entorhinal Cortex, DG: Dentate Gyrus

\begin{tabular}{|c|c|c|c|c|}
\hline Source Target & EC & DG & CA3 & CA1 \\
\hline EC & - & 0.1 & 0.06 & 0.06 \\
\hline DG & & - & 0.1 & \\
\hline CA3 & & & - & 0.1 \\
\hline CA1 & 0.1 & & & - \\
\hline
\end{tabular}

CA1 ([Andersen et al., 2007]). The connection probability of any two neurons from different regions is summed up in Table 5.

On the other hand, and as was also done in [Taxidis et al., 2012], the connection probability $p$ between any two neurons within the same region depends on the distance $D$ between them, following a gaussian-like distribution : $p=A \cdot e^{-\frac{D^{2}}{2 \sigma^{2}}}$. The width $\sigma$ of this distribution was set to $2500 \mu m$ for pyramidal cells, in accordance with [Ropireddy et al., 2011] (which states that hippocampal pyramidal cells make half of their connections within $1.3-1.7 \mathrm{~mm}$ ), and to the value of $350 \mu \mathrm{m}$ for interneurons, in accordance with the values reported in [Freund and Buzsáki, 1996] for hippocampal basket cells (which states that the transverse extent of their axon is between 900 and $1300 \mu \mathrm{m}$ ). The connection probability distributions for each region are then characterized by the probability $A$ of two neurons within a distance $D$ close to zero to be connected, summed up in Table 6 (Sources : [Couey et al., 2013], [Patton and McNaughton, 1995], [Taxidis et al., 2012], [Larimer and Strowbridge, 2008], [Debanne et al., 1995], [Knowles and Schwartzkroin, 1981]. Note that parameters come from experiments on human or rat hippocampus depending on the availability of the data). 
Table 6 Maximum probability $A$ of the synaptic connectivity within each region. EC: Entorhinal Cortex, DG: Dentate Gyrus. Py: Pyramidal neuron, In: Interneuron (the first two letters indicates the presynaptic neuron type while the second one indicates the post-synaptic neuron type).

\begin{tabular}{|c|c|c|c|c|}
\hline & Py-Py & Py-In & In-Py & In-In \\
\hline EC & 0 & 0.37 & 0.54 & 0 \\
\hline DG & 0 & 0.06 & 0.14 & 0 \\
\hline CA3 & 0.56 & 0.75 & 0.75 & 0 \\
\hline CA1 & 0 & 0.28 & 0.3 & 0.7 \\
\hline
\end{tabular}

Note that the synaptic connectivity within and between different structures of our network is concordant with the two-step memory process described by Hasselmo in [Hasselmo, 1999] for wakefulness and slow-wave sleep.

\subsubsection{Network input}

In order to represent inputs from the rest of the brain, each of the entorhinal cortex neurons is driven by a Poisson input whose variable firing rate is drawn from the envelope of the real sleep/wake SEEG signals measured in the prefrontal cortex, the lateral temporal lobe and the temporal pole.

More precisely, three groups of 10000 Poisson neurons were defined : each of their firing rates was obtained by first high-pass filtering the LFP from one of these regions above $5 \mathrm{~Hz}$, then taking its absolute value, and finally scaling it to a $0-200 \mathrm{~Hz}$ range. Each of the Poisson neuron group was connected to the entorhinal cortex region with a uniform probability of 0.01 through excitatory synapses with the same conductance as above.

The inputs to the network are later referred to as "wakefulness inputs" or as "slow-wave sleep inputs" (or shortly "sleep inputs") depending on whether SEEG signals from awake or deeply sleeping states were used to compute the input of the network.

\subsubsection{Network output: extracellular recordings}

The analysed output is the extracellular potential generated by the network, both at microscopic and at macroscopic scales.

The modelling of the LFP follows the approach proposed in [Mazzoni et al., 2015]. More precisely, the potential in every point in space was approximated by a weighted sum of the synaptic currents arriving at each pyramidal neuron, and neglecting the influence of the interneurons. Considering the neurons as dipoles, the contribution of a neuron of length $L$ to the extracellular potential at any point in space, at a distance $r$ and an angle $\theta$ from the midpoint of the neuron (see Figure 3), writes as:

$$
U=\frac{L \cos \theta}{4 \pi \sigma r^{2}}\left(I_{s y n_{E}}+I_{s y n_{I}}\right)
$$

where $\sigma=0.3 \mathrm{~S} / \mathrm{m}$ is the conductivity of the extracellular medium, which we considered homogeneous.

The LFP at one point is the sum of the contributions from all the pyramidal neurons in the entorhinal cortex and the hippocampus (dentate gyrus, CA3, and 


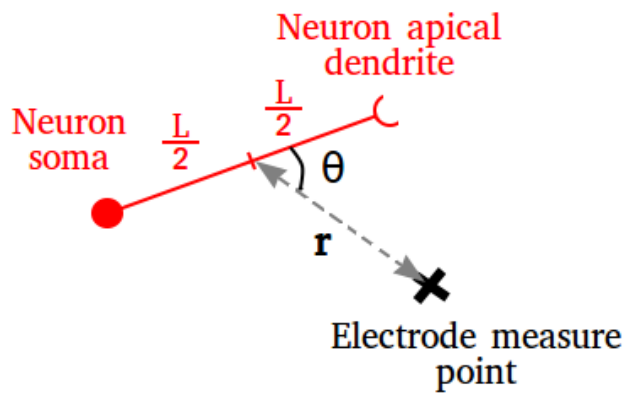

Fig. 3 Diagram of the parameters $L, r$ and $\theta$ used to compute the contribution of single neuron to the LFP.

CA1). In order to model the signal recorded by the macroscopic electrode and to compare it with real patient recordings, we averaged the LFP on two sets of 144 points evenly distributed on a cylinder of diameter $0.8 \mathrm{~mm}$ going through the network, each of them representing a $2 \mathrm{~mm}$-long contact, separated by $1.5 \mathrm{~mm}$ (as was also done in [Cosandier-Rimele et al., 2007]), and computed the difference between the two resulting signals (see figure 2).

Similarly to what is done by our intracranial EEG recording system, the simulated LFP was also bandpass filtered between $0.15 \mathrm{~Hz}$ and $480 \mathrm{~Hz}$ and downsampled to $1024 \mathrm{~Hz}$.

\subsection{Simulation}

The aim of this work is to test three main hypotheses for explaining the sleep/wake variations of the signals recorded inside the human hippocampal formation, two of which are directly linked to the concentration of Ach in the system.

1. The role of the synaptic connectivity: The varying concentration of Ach between wakefulness and slow-wave sleep modulate the synaptic interactions between and within hippocampal subregions. According to [Hasselmo, 1999], high Ach concentration during wakefulness reduces synaptic interactions from CA3 to CA1 and back to the entorhinal cortex, thus promoting short-term memory storage in CA3, and conversely the low concentration of Ach during sleep strengthen these connections and promotes long-term memory formation in the neocortex. More precisely, due to the presence of nicotinic and muscarinic receptors on hippocampal neurons, it has been shown that some synaptic connections are enhanced by a high Ach concentration, such as the inhibitory connections within the dentate gyrus and CA1 ([Jones and Yakel, 1997], [Frazier et al., 1998]), or the excitatory connections from the dentate gyrus ([Cheng 

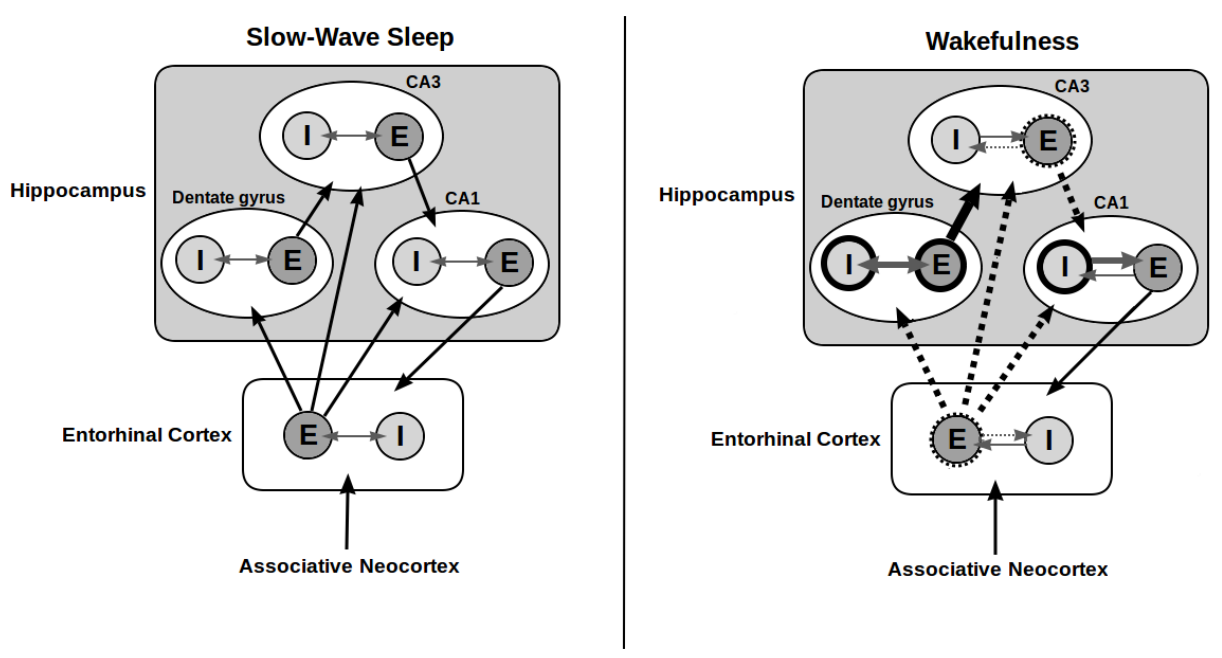

Fig. 4 Synaptic connectivity for slow-wave sleep and wakefulness. Bold lines indicate increased connectivity, and dotted lines indicate decreased connectivity during wakefulness compared to slow-wave sleep

and Yakel, 2013], [Gray et al., 1996]), while others are reduced, such as the excitatory connections from the entorhinal cortex ([Heys et al., 2012]) or CA3 ([Herreras et al., 1988]). In our simulations, after having tested different sets of parameters, we chose to model this effect by multiplying or dividing by a factor $f=3$ the conductance $g_{\max _{E}}$ or $g_{\max _{I}}$ of these connections to represent higher Ach concentration during wakefulness. These two connectivity settings, summed up in Figure 4, will be called later on in this article "slow-wave sleep connectivity" and "wakefulness connectivity". It is worth noting that other biological mechanisms apart from the varying concentration of Ach could also cause changes in the functional connectivity during the sleep-wake cycle, such as synaptic plasticity ([Tononi and Cirelli, 2006]) or differential activity of distinct interneurons subtypes ([Gan et al., 2017]).

2. The role of CAN currents : As presented in [Yoshida et al., 2012], Ach also plays a key role in the activation of CAN currents in hippocampal pyramidal cells. To account for the low concentration of Ach during slow-wave sleep, we chose to divide by 50 the maximum conductance $g_{C A N}$ of this channel in the corresponding simulations.

3. The role of the input : The last possible factor inducing sleep wake variations that we are investigating here is the (glutamatergic) input of the hippocampal network. Indeed, the inputs the hippocampal formation receives from its afferent structures also vary between wakefulness and sleep, as the whole brain exhibits different oscillatory rhythms. Therefore we chose to change the input stimulation to our network accordingly, by basing it on measured LFP signals measured in the patient's brain either during wakefulness or slow-wave sleep (see Section 2.2.5). Please note that cholinergic inputs (from the medial septum for example) are only represented by changing the two other settings (functional connectivity and CAN currents), and that other non-glutamatergic inputs are not directly modeled here. 
All the simulations were performed using the Brian2 libraries for Python ([Stimberg et al., 2014]), on the Grid'5000 testbed, supported by a scientific interest group hosted by Inria and including CNRS, RENATER and several Universities as well as other organizations (see https://www.grid5000.fr).

\subsubsection{Analysis of the real and simulated signals}

Meaningful events were located in the simulated signals with a method close to that of ([Taxidis et al., 2012]). First, the root mean square (RMS) of the signal was computed using 10ms-long time windows with no overlap, and its standard deviation (SD) was derived from its whole length. Portions of signal where the RMS was greater than $2 \mathrm{xSD}$, with a peak above $4 \mathrm{xSD}$, were considered as events.

The spectrum of all the events was then analyzed to determine their peak frequency and to identify the type of oscillatory patterns: in the following sections, all filters applied to the signals are Butterworth filters of order 2.

The distribution of the peak frequencies in the different simulations performed were compared using WilcoxonMannWhitney tests (with the purpose of avoiding making assumptions on the shape of the distributions).

In the real SEEG signals, a similar procedure was used to detect SWR, except that the RMS was computed from the $120-250 \mathrm{~Hz}$ bandpass filtered signal and only events when the peak RMS was above $6 \mathrm{xSD}$ were considered.

\section{Results}

\subsection{Network Topology influences the measured LFP}

One of the original contributions of this work is the use of an approximation of the real anatomy of the simulated systems. Therefore, regardless and previous to any sleep/wake analysis, one of our first concerns was to check what could be the benefits of using a anatomically realistic topology and connectivity compared to, say, a random one.

Figure 5 and Figure 6 present the simulated LFP with slow-wave sleep and wakefulness parameters respectively in 3 different networks: a first network with topology and connectivity as defined in Section 2, a second network with the same topology but a uniform rather than distance-related connectivity, and a third network where the connectivity was kept but where each structure's topology was replaced with a simple rectangular-shaped column. Other parameters were kept the same across simulations.

What can be seen is that both topology and connectivity influence the results of the simulations for slow-wave sleep parameters. Sharp-wave ripples-like events (see Section 3.2) can only be seen with a distance-related connectivity and not with uniform connectivity. The events obtained with the full topology are closer to the frequency of sharp-wave ripples reported in the literature (see [Buzsáki, 2015]), while those obtained with a simplified topology lie within the gamma band (and there is proportionally more power in the theta and gamma bands compared to the ripple band in this case). 

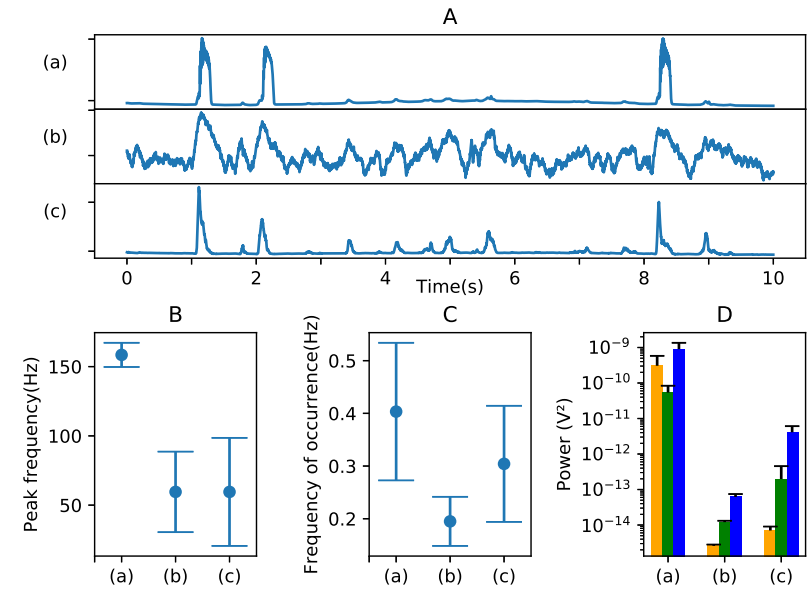

Fig. 5 Comparison of the simulated LFP for slow-wave sleep, with the following topology and connectivity : (a) full topology and distance-related connectivity, (b) full topology but uniform connectivity, and (c) simplified topology and distance-related connectivity. A: 10-second LFP traces for each conformation. B: Peak frequency of the detected events in each LFP simulations, for each conformation, with mean and SD. Statistical comparison shows significant difference between each case $\left(p<10^{-3}\right)$. C: Frequency of occurrence of the events for each conformation, with mean and SD. D: Power in in the oscillations in the Ripple (120-200Hz, yellow), Gamma $(30-100 \mathrm{~Hz}$, green) and Theta $(5-10 \mathrm{~Hz}$, blue) frequency bands, with mean and SD, for each conformation.

On the other hand, theta and gamma oscillations can be obtained with all the three networks, though the temporal aspect of the simulated signal is modified by changing either the connectivity or the topology.

A possible explanation as for why the topology and connectivity can affect so much the resulting simulations in the slow-wave sleep case is that it changes the number and sizes of interconnected neuronal clusters and therefore without a detailed topology it is hard to get the same kind of neural synchronization as seen during sharp-wave ripple complexes. To a lesser extent, changing the position of the neurons and their orientation when changing the topology also affects the resulting signal as it affects the way neural activities are summed up when computing the LFP seen by the electrode.

\subsection{Sharp Wave-Ripples complexes appear with Slow-Wave Sleep parameters}

With slow-wave sleep connectivity, slow-wave sleep inputs, and reduced CAN currents, large amplitude events can be seen on the simulated electrode every few seconds (11 events in a typical one-minute-long simulation, with mean duration $156 \mathrm{~ms})$, as the one shown on figure 7 . Band-pass filtering them between 30 and $400 \mathrm{~Hz}$ reveals an oscillatory pattern with peak frequency at $161 \mathrm{~Hz}$ (SD: $9 \mathrm{~Hz}$ ) (see also Figure 13), which is consistent with the literature on sharp-wave ripple complexes for in vivo recordings ([Buzsáki, 2015]).

The ripples in the simulation also appear to happen with a timing close to that of the sharp-waves ripples in the LFP recorded in the patient's hippocampus, as 

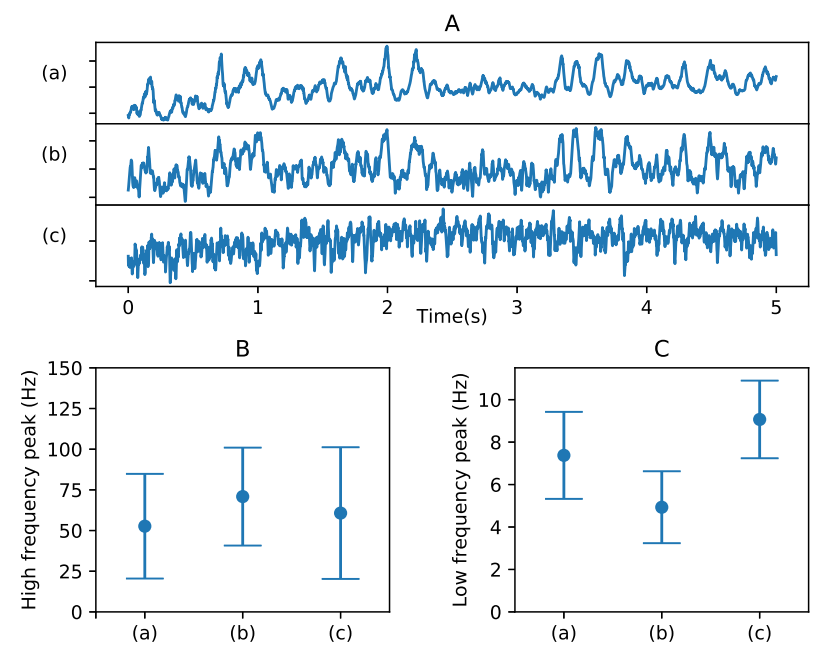

Fig. 6 Comparison of the simulated LFP for wakefulness, with the following topology and connectivity : (a) full topology and distance-related connectivity, (b) full topology but uniform connectivity, and (c) simplified topology and distance-related connectivity. A: 5-second LFP traces for each conformation. B: Peak frequency of the detected events in each LFP simulations, for each conformation, with mean and SD. Statistical comparison shows significant difference between each case $\left(p<10^{-3}\right)$. C: Peak frequency of the LFP filtered in the theta band (5$10 \mathrm{~Hz}$ ), for each conformation, with mean and SD.

shown in Figure 8. In ten one-minute-long signals, 228 SWR were found in the measured LFP and 235 in the corresponding simulations. $62 \%$ of the simulated events were located within 1 second of a SWR in the corresponding measured signal, the simulated SWRs occurring in average $220 \mathrm{~ms}$ before a measured SWR (SD:1.8s).

The raster plot and population coherence measure of the different regions reveals that the sharp-wave ripples seen on the LFP come from a synchronized activity of CA1 pyramidal cells that is initiated by a strong inhibitory input from the interneurons in CA3 (followed by excitation from CA3 and then strong inhibition in CA1 itself). During the ripple, interneurons in CA1 and CA3 are relatively silent, and only get strongly synchronized again around 100ms later, possibly causing the termination of the ripple (this inhibitory activity originates from the Dentate Gyrus). This activity is similar to what has been reported for O-LM interneurons in particular (see [Somogyi et al., 2014]). The evolution of a coherence measure of the CA3 and CA1 regions during a ripple (computed as in [Wang and Buzsáki, 1996] on sliding windows of width $50 \mathrm{~ms}$ with $75 \%$ overlap) is shown on Figure 9.

However, almost all of the CA1 pyramidal neurons fire during each of these events, therefore it does not fully respect what was reported in the literature, as in [Nádasdy et al., 1999]. The direction of the propagation of the ripples in CA1, as well as their original location, varies between events (figure not shown), in accordance with [Patel et al., 2013].

The spectrum of the simulated signal is quite similar to that of the SEEG signal measured in the hippocampus during slow-wave sleep, though the simulated signal shows a stronger power density in the ripple frequency band (see Figure 12). This 

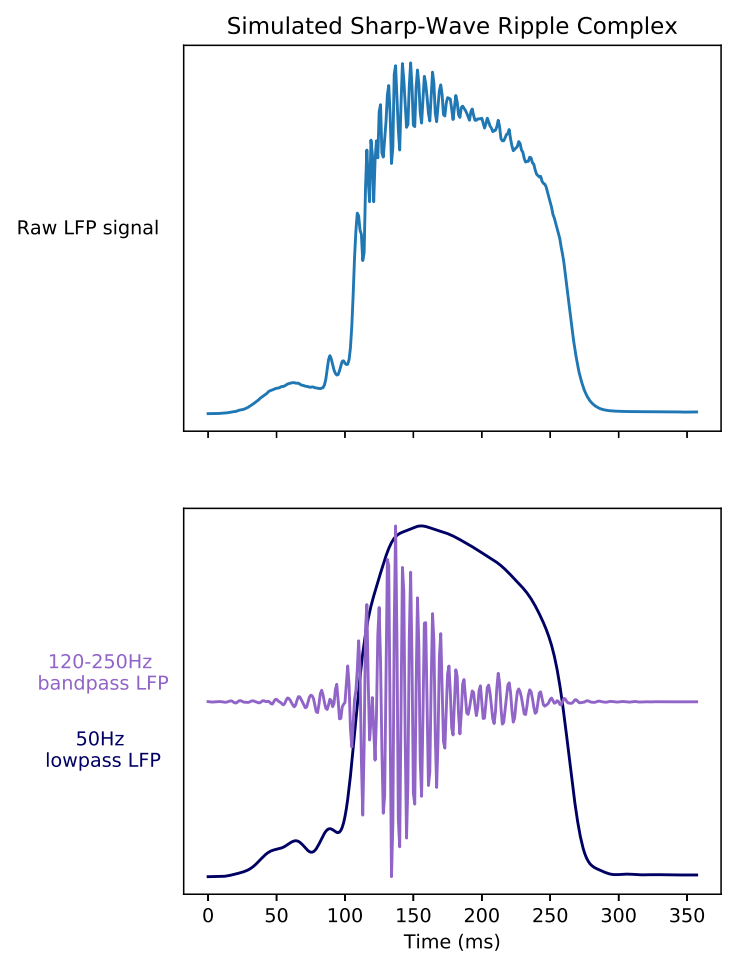

Fig. 7 LFP simulated at the electrode during a ripple, and corresponding $120-250 \mathrm{~Hz}$ bandpass filtered and $50 \mathrm{~Hz}$ lowpass filtered signals.

could be explained by the fact that our simulated neurons only model a small layer of the hippocampus where CA1 pyramidal cells almost all get involved in the SWRs and get highly synchronized, and therefore our simulations don't show enough noise and power in other frequency bands. Also, we do not include in our simulation the LFP generated by neighboring structures and propagated through the extracellular medium, which could increase the energy in the low frequencies of the LFP and therefore could compensate for the apparent high frequency peak that we observe in our simulations.

\subsection{Theta-nested Gamma Oscillations appear with Wakefulness parameters}

Switching the network parameters to the wakefulness ones gives rise to different oscillatory rhythms. Indeed, when applying a similar method of event detection as for slow-wave sleep, that is when looking for portions of the signal with RMS significantly above its standard deviation (see Section 2.3.1), it can be seen that the events' peak frequency is shifted towards the gamma range $(53 \mathrm{~Hz}, \mathrm{SD} 32 \mathrm{~Hz})$. 

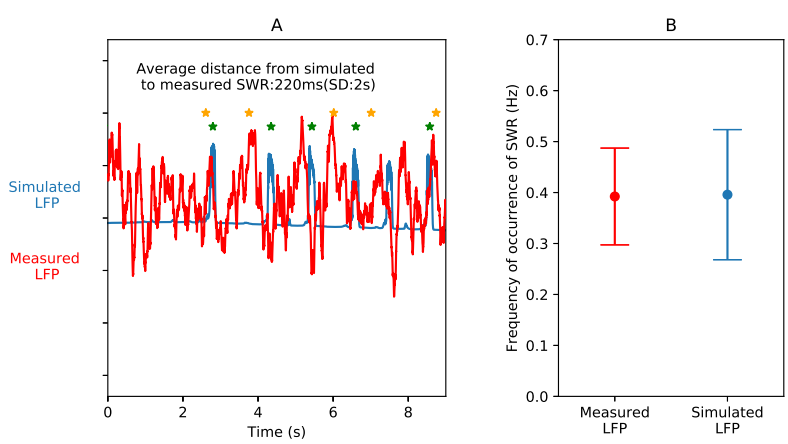

Fig. 8 A : Comparison between the position of sharp-wave ripples in the simulation (red stars) and in the slow-wave sleep recordings of the hippocampus (green stars). The blue line corresponds to the simulated LFP, and the red line to the recorded one. B : Frequency of occurrence of the sharp-wave ripples in the measured and simulated LFP.

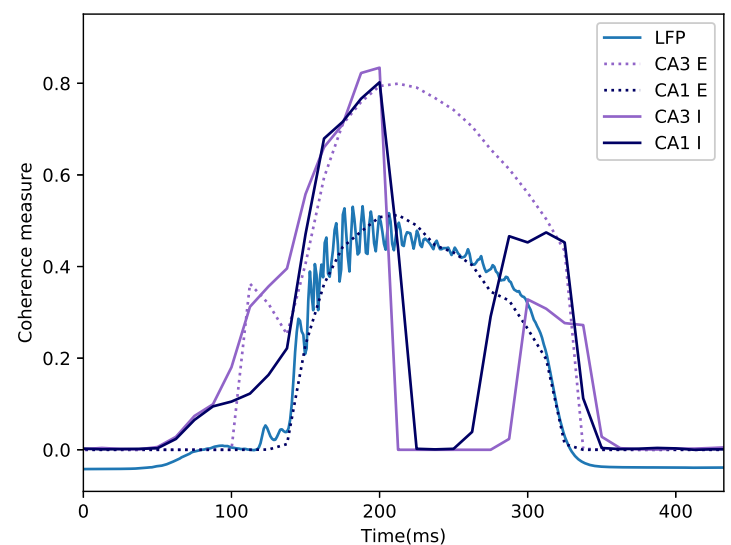

Fig. 9 Population coherence plots in the CA3 and CA1 regions for excitatory neurons (E, dotted lines) and inhibitory neurons (I, solid lines) during a ripple. In light blue, the corresponding normalized simulated LFP is shown.

Also, the signal now presents lower frequency oscillations, with a peak in its power spectral density around $7 \mathrm{~Hz}$, which corresponds to the theta frequency band (see Figure 10).

More precisely, power in the gamma frequency band $(30-100 \mathrm{~Hz})$ is mostly located around the peak of the theta oscillations (see Figure 11). On the other hand, the frequency of the gamma oscillations doesn't change significantly depending on the phase of the theta oscillations.

The spectrum of the simulated signal is similar to that of the measured SEEG in the hippocampus (see Figure 12), though it has more power in the theta frequency band, possibly for the same reasons as the one explained earlier in Section 3.2. 

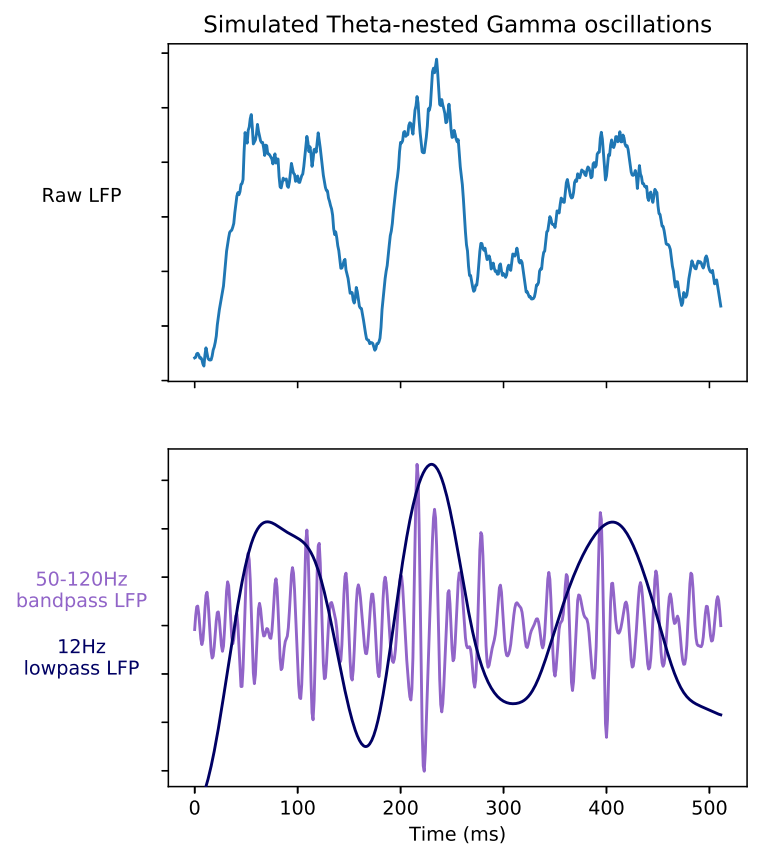

Fig. 10 LFP simulated at the electrode during theta-nested gamma oscillations, and corresponding $50-120 \mathrm{~Hz}$ bandpass filtered and $12 \mathrm{~Hz}$ lowpass filtered signals.

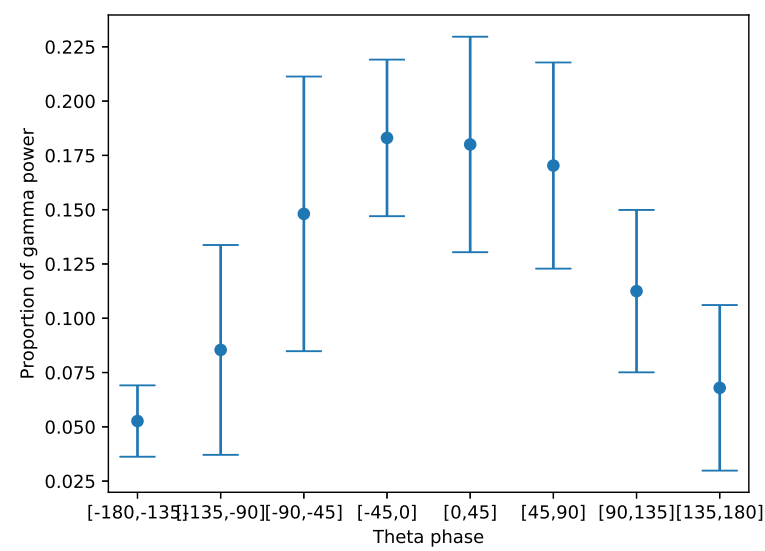

Fig. 11 Proportion of the power in the gamma frequency band depending on the phase of the theta oscillations, in ten one-minute-long simulations 

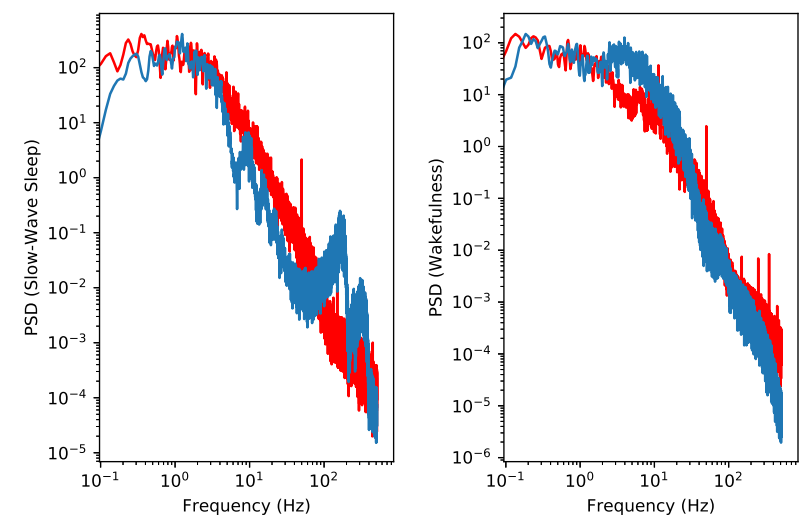

Fig. 12 Comparison between the power spectrum of the hippocampal LFP (blue) and the corresponding simulated signal (orange), during slow-wave sleep (left) and wakefulness (right).

\subsection{Factors influencing the sleep-wake oscillatory rhythms}

After having confirmed that slow-wave sleep and wakefulness parameters (input, CAN current and functional connectivity) were associated with sharp-wave ripples and theta-nested gamma oscillations respectively, we tried to modify each of them individually to analyze their influence. Note that among these parameters, two are continuously varying (functional connectivity gain $f$ and CAN channel conductance $g_{C A N}$, while the third one is binary (sleep/wake input).

A first analysis can actually be done by binarizing all three factors. As we have determined the most plausible values that generate either sleep rhythms (i.e. SWR) or wake rhythms (theta-gamma oscillations), we can choose the respective values for the connectivity gain and the CAN conductance $\left(\mathrm{f}=\{1,3\}\right.$ and $g_{C A N}=$ $\{0.5,25\} \mu S)$. These values are designated in the following by sleep $\left(f=1, g_{C A N}=\right.$ $0.5)$ and wake $\left(f=3, g_{C A N}=25\right)$ respectively.

As it can be seen in Figures 13, the high frequency component of the oscillations depends mostly on the functional connectivity, with little effect from the CAN current and almost no effect from the input: slow-wave sleep connectivity produces events within ripple frequency range, while wakefulness connectivity produces peak frequencies within the gamma band. The input type (sleep/wake) does not significantly change these peak frequencies. The CAN conductance has a slightly more important effect, sufficient for changing the peak frequency when the connectivity is low (sleep connectivity). Similar results can also be seen on Figure 14, as whenever the connectivity is set to slow-wave sleep, there is more power in the ripple band than in the gamma band and conversely with wakefulness connectivity. The CAN currents increase the overall power in the signal in every parameter set, and also increase the frequency of occurrence of SWRs seen with slow-wave sleep connectivity, from $0.3 \mathrm{~Hz}(\mathrm{SD} 0.1 \mathrm{~Hz})$ to $1.6 \mathrm{~Hz}(\mathrm{SD} 0.1 \mathrm{~Hz})$ with sleep inputs and from $0.13 \mathrm{~Hz}(\mathrm{SD} 0.04 \mathrm{~Hz})$ to $1.25 \mathrm{~Hz}(\mathrm{SD} 0.4 \mathrm{~Hz})$ with wakefulness inputs (in each case the p-value is less than 0.01). The power of the signals are much higher with slow-wave sleep connectivity than with wakefulness connectivity, 
which may be due to the very high neuronal activity seen during our simulated SWRs.

In order to asses more precisely the effect of the CAN conductance variations, we have also modified it gradually between the two extreme values (see Figure 17, left panel; recall that the on/off CAN current labels in Figure 13 correspond to the most extreme cases in Figure 17).

Figure 13 indicates that functional connectivity is the most important factor for changing the peak frequency. The natural question which arises is if this change in the peak frequency is gradual when we vary the connectivity gain $f$ or if there is a switch at some point. This is explored in Figure 15 (left panel). As it can be seen, the peak frequency varies rather continuously with the connectivity, but nonlinearly. Small values of $f$ ensure the generation of ripples, while values greater than 1.5 lower the peak frequencies into the gamma band. For conciseness, we present here the dependency of the peak frequency with $f$ for fixed values of the CAN conductance (to one of the extreme values, namely wake) and the input (wake also), but the results are similar for other combinations.

The low frequency part of the simulated signals seems to be influenced differently by these three factors. Indeed, low-passing the signals below $20 \mathrm{~Hz}$ and looking at the highest frequency peak in their spectrum shows that the low frequency component of the oscillations seems to be influenced mostly by the CAN current, with smaller effect from the input or the connectivity (see Figure 16). High CAN current $\left(g_{C A N} \geq 25 \mu \mathrm{S} / \mathrm{cm}^{2}\right.$, see Figure 17, right panel) is a sufficient condition for the oscillations to reach the theta band. Similar results can be obtained even if the CAN current is high only in the CA1 and CA3 neuron populations and not in the entorhinal cortex. On the contrary, varying the functional connectivity alone (while maintaining the input and the CAN conductance in wake states) does not significantly alter the low frequency part of the simulated signals (see Figure 15, right panel). Still, theta oscillations can also be generated in the absence of a high CAN current (which thus is not a necessary condition), if both connectivity and input are in the wake state. If one of them (or both) are in the sleep state, the low frequency part remains below the inferior bound of the theta band.

\section{Discussion}

In this work, we built a computational model of the hippocampus capable of reproducing both slow-wave sleep sharp-wave ripples and wakefulness theta-nested gamma oscillations. This models takes advantage of a realistic network topology and synaptic connectivity, and includes effects on individual neurons and functional connectivity that are coherent with the varying concentration of Acetylcholine over the sleep-wake cycle. Overall, it is consistent with the theory from [Hasselmo, 1999] stating that Ach could be an important factor in the changes of rhythms observed in the hippocampus in the context of the two-stage model of memory formation. However it should be noted that other mechanisms might be at work than Ach modulation, leading to similar effects (especially regarding the synaptic connectivity).

Our results suggest that the high frequency component of hippocampal oscillations might be governed mostly by network connectivity, whereas the low frequency components could result from individual neuron channel properties. A 


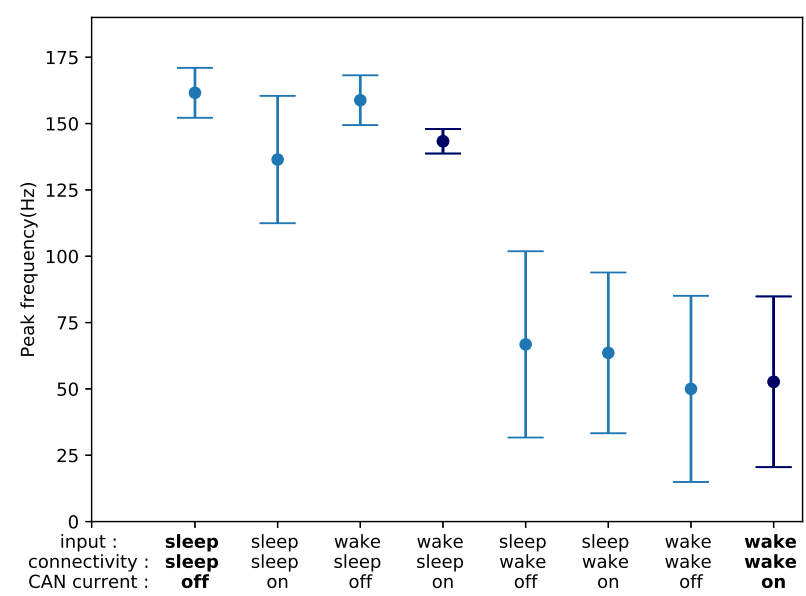

Fig. 13 Distribution of the peak frequencies of events in ten one-minute-long simulations with Sleep or Wakefulness stimulation, Sleep or Wakefulness connectivity, and presence or absence of $\mathrm{CAN}$ current, with mean and standard deviation ( $\mathrm{N}$ indicates the number of events). Whenever a different connectivity is used, the peak frequency is significantly different $\left(p<10^{-3}\right)$. The number of studied events in each case is $\mathrm{N}=234,967,76,750,309,218,164$ and 272 respectively. The dark blue points correspond to the same set of parameters chosen for the study of the influence of the connectivity in Fig 15.

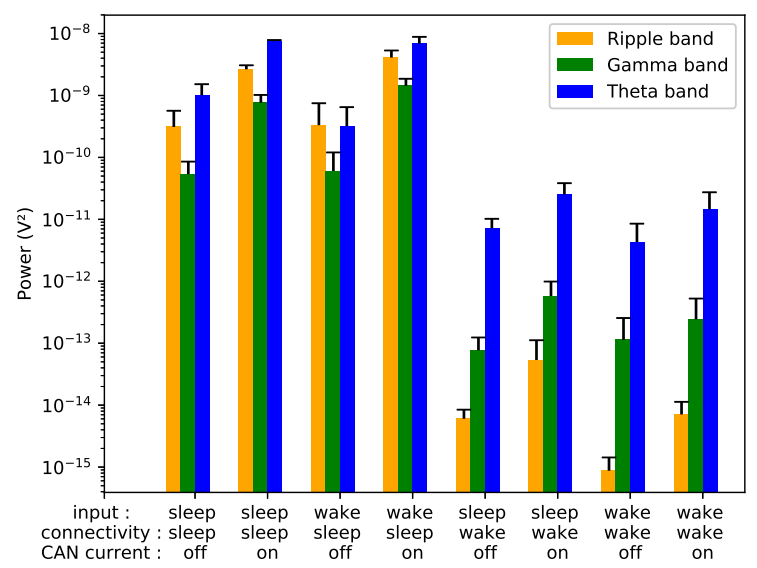

Fig. 14 Power in the oscillations in the Ripple (120-200Hz, yellow), Gamma (30-100Hz, green) and Theta $(5-10 \mathrm{~Hz}$, blue) frequency bands in ten one-minute-long simulations with Sleep or Wakefulness stimulation, Sleep or Wakefulness connectivity, and presence or absence of CAN current, with mean and standard deviation. 

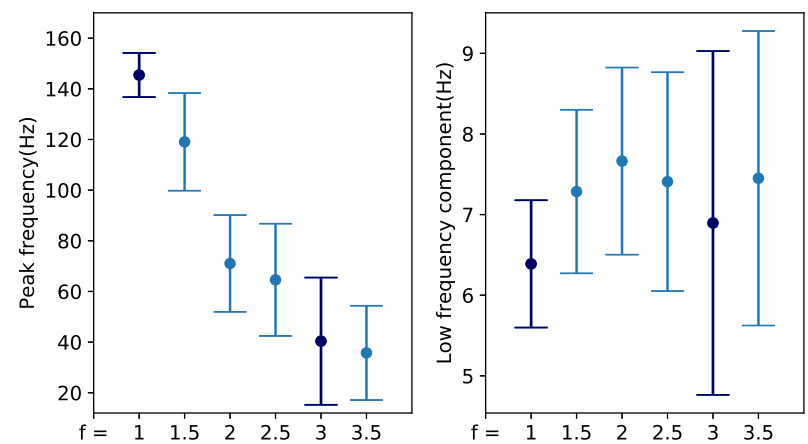

Fig. 15 Influence of the functional connectivity modification factor $f$, with wakefulness input and high CAN current. In dark blue, the value $f=1$ corresponds to the sleep connectivity and $f=3$ corresponds to the wakefulness one. Left: Distribution of the peak frequencies of events. Right: Distribution of the low frequency component of the signals (ten simulations in each case).

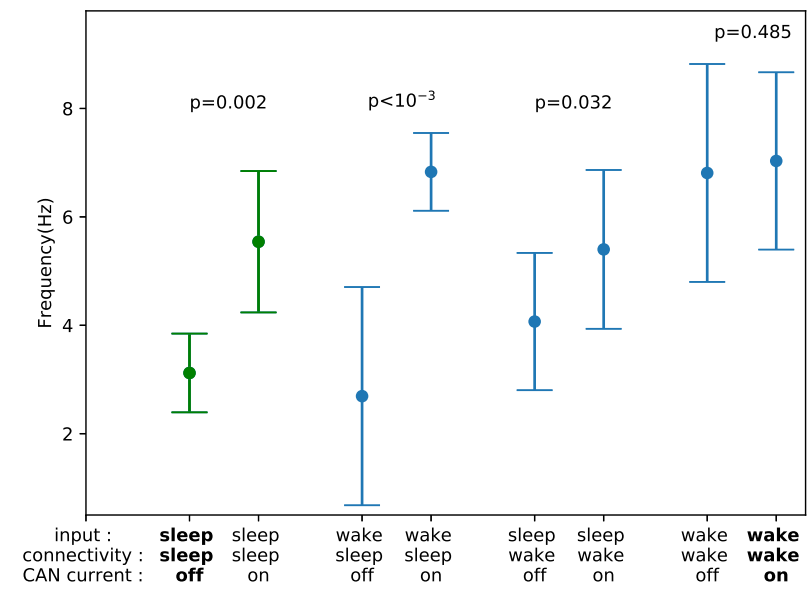

Fig. 16 Distribution of the low frequency components of the neural oscillations in ten oneminute-long simulations with Sleep or Wakefulness stimulation, Sleep or Wakefulness connectivity, and presence or absence of CAN current. The p-values indicated correspond to the comparison of the signals with and without CAN in each situation. With CAN current, the oscillations lie within the theta frequency band. The green points correspond to the same set of parameters chosen for the study of the influence of the CAN channel conductance as in Fig 17.

possible consequence of this idea is that higher frequency, pathological oscillations that are seen in the epileptic hippocampus for example (fast ripples or seizures) might also emerge from an abnormal functional connectivity of the system rather than individual neuron properties.

An interesting point to raise is that the glutamatergic inputs given to the network seemed to have little to no influence on the frequency of our model's oscillations, which could indicate that the hippocampus doesn't need an external "pacemaker" such as the medial septum to set its rhythms, but it has its own 

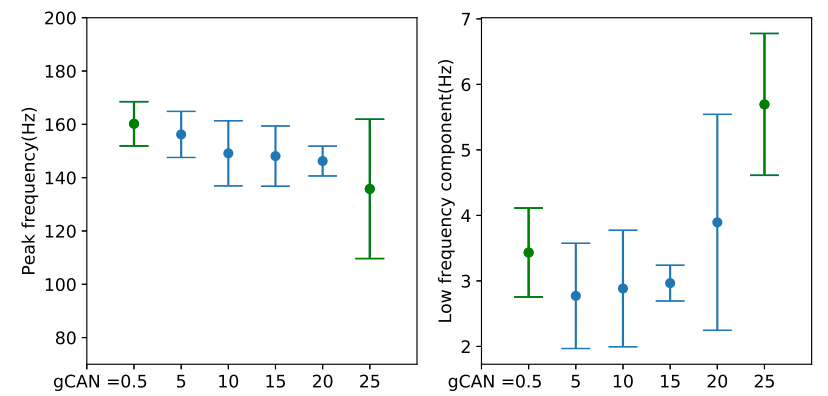

Fig. 17 Influence of the CAN channel conductance $g_{C A N}$ (in $\mu S / \mathrm{cm}^{2}$ ). In green, the value $g_{C A N}=0.5 \mu S / \mathrm{cm}^{2}$ corresponds to slow-wave sleep settings and $g_{C A N}=25 \mu \mathrm{S} / \mathrm{cm}^{2}$ corresponds to wakefulness. Left: Distribution of the peak frequencies of events. Right: Distribution of the low frequency component of the signals (ten simulations in each case).

internal pacemaker, the CAN neurons (for more arguments on that topic, see for example [Hangya et al., 2009], [Kang et al., 2015] or [Buzsáki, 2002]). Still, the external influence cannot be excluded, as our model predicts that theta oscillations can also be generated by the correct combination of the inputs and functional connectivity, as mentioned in the previous section. On the other hand, the inputs seem to influence the timing at which oscillations are generated, which could account for signal encoding, particularly during wakefulness. On portions of the signals, the simulation even showed some similarities with the measurements in the temporal domain and not only in the frequency domain (see Figure 8).

Similarly to what have been proposed by [Taxidis et al., 2012], our model was able to reproduce different rhythms, and sharp-wave ripples in particular, by using only chemical synapses (and therefore no axo-axonic gap junctions). This further supports the idea that gap junctions may not be necessary for generating high frequency oscillations in the hippocampus.

Finally, our work suggests that the topology of the hippocampal formation should be taken into account in order to improve existing computational models. In particular, such methods could produce a realistic SEEG signal estimation while keeping the simulation complexity low, by solving the differential equations for point neurons only and considering them as dipoles for calculating local field potentials.

\section{Information Sharing Statement}

All the Python source files used for building the network and running the simulations are accessible on the ModelDB public repositories.

\section{References}

[Andersen et al., 2007] Andersen, P., Morris, R., Amaral, D., Bliss, T., and O'Keefe, J. (2007). The hippocampus book. Oxford University Press.

[Axmacher et al., 2010] Axmacher, N., Henseler, M. M., Jensen, O., Weinreich, I., Elger, C. E., and Fell, J. (2010). Cross-frequency coupling supports multi-item working memory in the human hippocampus. Proceedings of the National Academy of Sciences, 107(7):3228-3233. 
[Bartos et al., 2007] Bartos, M., Vida, I., and Jonas, P. (2007). Synaptic mechanisms of synchronized gamma oscillations in inhibitory interneuron networks. Nature reviews. Neuroscience, 8(1):45-56.

[Buzsáki, 2002] Buzsáki, G. (2002). Theta oscillations in the hippocampus. Neuron, 33(3):325 -340 .

[Buzsáki, 2015] Buzsáki, G. (2015). Hippocampal sharp wave-ripple: A cognitive biomarker for episodic memory and planning. Hippocampus, 25(10):1073-1188.

[Cheng and Yakel, 2013] Cheng, Q. and Yakel, J. L. (2013). Presynaptic $\alpha 7$ nicotinic acetylcholine receptors enhance hippocampal mossy fiber glutamatergic transmission via pka activation. Journal of Neuroscience, 34(1):124-133.

[Cosandier-Rimele et al., 2007] Cosandier-Rimele, D., Badier, J., Chauvel, P., and Wendling, F. (2007). A physiologically plausible spatio-temporal model for eeg signals recorded with intracerebral electrodes in human partial epilepsy. IEEE Transactions on Biomedical Engineering, 54(3):380-388.

[Couey et al., 2013] Couey, J. J., Witoelar, A., Zhang, S.-J., Zheng, K., Ye, J., Dunn, B., Czajkowski, R., Moser, M.-B., Moser, E. I., Roudi, Y., et al. (2013). Recurrent inhibitory circuitry as a mechanism for grid formation. Nature neuroscience, 16(3):318-324.

[Debanne et al., 1995] Debanne, D., Guerineau, N. C., Gahwiler, B. H., and Thompson, S. M. (1995). Physiology and pharmacology of unitary synaptic connections between pairs of cells in areas ca3 and ca1 of rat hippocampal slice cultures. Journal of Neurophysiology, 73(3):1282-1294. PMID: 7608771.

[Drever et al., 2011] Drever, B. D., Riedel, G., and Platt, B. (2011). The cholinergic system and hippocampal plasticity. Behavioural Brain Research, 221(2):505 - 514. The cholinergic system and brain function.

[Foster and Wilson, 2006] Foster, D. J. and Wilson, M. A. (2006). Reverse replay of behavioural sequences in hippocampal place cells during the awake state. Nature, 440(7084):680.

[Frazier et al., 1998] Frazier, C. J., Rollins, Y. D., Breese, C. R., Leonard, S., Freedman, R., and Dunwiddie, T. V. (1998). Acetylcholine activates an $\alpha$-bungarotoxin-sensitive nicotinic current in rat hippocampal interneurons, but not pyramidal cells. Journal of Neuroscience, 18(4):1187-1195.

[Freund and Buzsáki, 1996] Freund, T. and Buzsáki, G. (1996). Interneurons of the hippocampus. Hippocampus, 6(4):347-470.

[Fukai, 1999] Fukai, T. (1999). Sequence generation in arbitrary temporal patterns from thetanested gamma oscillations: a model of the basal gangliathalamo-cortical loops. Neural Networks, 12(78):975 - 987 .

[Gan et al., 2017] Gan, J., ming Weng, S., Perna-Andrade, A. J., Csicsvari, J., and Jonas, P. (2017). Phase-locked inhibition, but not excitation, underlies hippocampal ripple oscillations in awake mice invivo. Neuron, 93(2):308 - 314.

[Giovannini et al., 2017] Giovannini, F., Knauer, B., Yoshida, M., and Buhry, L. (2017). The can-in network: A biologically inspired model for self-sustained theta oscillations and memory maintenance in the hippocampus. Hippocampus, 27(4):450-463.

[Girardeau and Zugaro, 2011] Girardeau, G. and Zugaro, M. (2011). Hippocampal ripples and memory consolidation. Current Opinion in Neurobiology, 21(3):452 - 459. Behavioural and cognitive neuroscience.

[Gray et al., 1996] Gray, R., Rajan, A. S., Radcliffe, K. A., Yakehiro, M., and Dani, J. A. (1996). Hippocampal synaptic transmission enhanced by low concentrations of nicotine. Nature, 383(6602):713.

[Hangya et al., 2009] Hangya, B., Borhegyi, Z., Szilágyi, N., Freund, T. F., and Varga, V. (2009). Gabaergic neurons of the medial septum lead the hippocampal network during theta activity. Journal of Neuroscience, 29(25):8094-8102.

[Hasselmo, 1999] Hasselmo, M. E. (1999). Neuromodulation: acetylcholine and memory consolidation. Trends in Cognitive Sciences, 3(9):351 - 359.

[Herreras et al., 1988] Herreras, O., Sols, J., Herranz, A., del Ro, R. M., and Lerma, J. (1988). Sensory modulation of hippocampal transmission. ii. evidence for a cholinergic locus of inhibition in the schaffer-cal synapse. Brain Research, 461(2):303 - 313.

[Heys et al., 2012] Heys, J. G., Schultheiss, N. W., Shay, C. F., Tsuno, Y., and Hasselmo, M. E. (2012). Effects of acetylcholine on neuronal properties in entorhinal cortex. Frontiers in behavioral neuroscience, 6.

[Hodgkin and Huxley, 1952] Hodgkin, A. L. and Huxley, A. F. (1952). A quantitative description of membrane current and its application to conduction and excitation in nerve. The Journal of Physiology, 117(4):500-544. 
[Hofmanis et al., 2011] Hofmanis, J., Caspary, O., Louis-Dorr, V., and Maillard, L. (2011). Automatic depth electrode localization in intracranial space. In 4 th International Conference on Bio-inspired Systems and Signal Processing, Biosignals 2011, page CDROM, Rome, Italy.

[Jinno and Kosaka, 2010] Jinno, S. and Kosaka, T. (2010). Stereological estimation of numerical densities of glutamatergic principal neurons in the mouse hippocampus. Hippocampus, 20(7):829-840.

[Jones and Yakel, 1997] Jones, S. and Yakel, J. L. (1997). Functional nicotinic ach receptors on interneurones in the rat hippocampus. The Journal of Physiology, 504(3):603-610.

[Kang et al., 2015] Kang, D., Ding, M., Topchiy, I., Shifflett, L., and Kocsis, B. (2015). Theta-rhythmic drive between medial septum and hippocampus in slow-wave sleep and microarousal: a granger causality analysis. Journal of Neurophysiology, 114(5):2797-2803. PMID: 26354315.

[Knowles and Schwartzkroin, 1981] Knowles, W. and Schwartzkroin, P. (1981). Local circuit synaptic interactions in hippocampal brain slices. Journal of Neuroscience, 1(3):318-322.

[Larimer and Strowbridge, 2008] Larimer, P. and Strowbridge, B. W. (2008). Nonrandom local circuits in the dentate gyrus. Journal of Neuroscience, 28(47):12212-12223.

[Mazzoni et al., 2015] Mazzoni, A., Lindn, H., Cuntz, H., Lansner, A., Panzeri, S., and Einevoll, G. T. (2015). Computing the local field potential (lfp) from integrate-and-fire network models. PLOS Computational Biology, 11(12):1-38.

[Nádasdy et al., 1999] Nádasdy, Z., Hirase, H., Czurkó, A., Csicsvari, J., and Buzsáki, G. (1999). Replay and time compression of recurring spike sequences in the hippocampus. Journal of Neuroscience, 19(21):9497-9507.

[O'Keefe and Recce, 1993] O'Keefe, J. and Recce, M. L. (1993). Phase relationship between hippocampal place units and the eeg theta rhythm. Hippocampus, 3(3):317-330.

[Pastoll et al., 2013] Pastoll, H., Solanka, L., vanRossum, M., and Nolan, M. (2013). Feedback inhibition enables theta-nested gamma oscillations and grid firing fields. Neuron, 77(1):141 -154 .

[Patel et al., 2013] Patel, J., Schomburg, E. W., Berényi, A., Fujisawa, S., and Buzsáki, G. (2013). Local generation and propagation of ripples along the septotemporal axis of the hippocampus. Journal of Neuroscience, 33(43):17029-17041.

[Patton and McNaughton, 1995] Patton, P. E. and McNaughton, B. (1995). Connection matrix of the hippocampal formation: I. the dentate gyrus. Hippocampus, 5(4):245-286.

[Pettersen et al., 2012] Pettersen, K. H., Lindén, H., Dale, A. M., and Einevoll, G. T. (2012). Extracellular spikes and csd. Handbook of neural activity measurement, 1:92-135.

[Platt and Riedel, 2011] Platt, B. and Riedel, G. (2011). The cholinergic system, $\{$ EEG $\}$ and sleep. Behavioural Brain Research, 221(2):499 - 504. The cholinergic system and brain function.

[Ropireddy et al., 2011] Ropireddy, D., Scorcioni, R., Lasher, B., Buzsáki, G., and Ascoli, G. A. (2011). Axonal morphometry of hippocampal pyramidal neurons semi-automatically reconstructed after in vivo labeling in different ca3 locations. Brain Structure and Function, 216(1):1-15

[Ruivo et al., 2017] Ruivo, L. M. T.-G., Baker, K. L., Conway, M. W., Kinsley, P. J., Gilmour, G., Phillips, K. G., Isaac, J. T., Lowry, J. P., and Mellor, J. R. (2017). Coordinated acetylcholine release in prefrontal cortex and hippocampus is associated with arousal and reward on distinct timescales. Cell Reports, 18(4):905 - 917.

[Sarter et al., 2009] Sarter, M., Parikh, V., and Howe, W. M. (2009). Phasic acetylcholine release and the volume transmission hypothesis: time to move on. Nature Reviews Neuroscience, 10:383-390.

[Somogyi et al., 2014] Somogyi, P., Katona, L., Klausberger, T., Lasztóczi, B., and Viney, T. J. (2014). Temporal redistribution of inhibition over neuronal subcellular domains underlies state-dependent rhythmic change of excitability in the hippocampus. Philosophical Transactions of the Royal Society of London B: Biological Sciences, 369(1635).

[Stimberg et al., 2014] Stimberg, M., Goodman, D. F., Benichoux, V., and Brette, R. (2014). Equation-oriented specification of neural models for simulations. Frontiers in Neuroinformatics, 8.

[Taxidis et al., 2012] Taxidis, J., Coombes, S., Mason, R., and Owen, M. R. (2012). Modeling sharp wave-ripple complexes through a ca3-ca1 network model with chemical synapses. Hippocampus, 22(5):995-1017.

[Tiesinga et al., 2001] Tiesinga, P. H., Fellous, J.-M., Jos, J. V., and Sejnowski, T. J. (2001). Computational model of carbachol-induced delta, theta, and gamma oscillations in the hippocampus. Hippocampus, 11(3):251-274. 
[Tononi and Cirelli, 2006] Tononi, G. and Cirelli, C. (2006). Sleep function and synaptic homeostasis. Sleep Medicine Reviews, 10(1):49 - 62.

[Traub and Bibbig, 2000] Traub, R. D. and Bibbig, A. (2000). A model of high-frequency ripples in the hippocampus based on synaptic coupling plus axon-axon gap junctions between pyramidal neurons. Journal of Neuroscience, 20(6):2086-2093.

[Wang and Buzsáki, 1996] Wang, X.-J. and Buzsáki, G. (1996). Gamma oscillation by synaptic inhibition in a hippocampal interneuronal network model. Journal of Neuroscience, 16(20):6402-6413.

[West and Gundersen, 1990] West, M. J. and Gundersen, H. J. G. (1990). Unbiased stereological estimation of the number of neurons in the human hippocampus. The Journal of Comparative Neurology, 296(1):1-22.

[Yoshida et al., 2012] Yoshida, M., Knauer, B., and Jochems, A. (2012). Cholinergic modulation of the can current may adjust neural dynamics for active memory maintenance, spatial navigation and time-compressed replay. Frontiers in neural circuits, 6. 